\title{
Situational CUES TRIgger Risk ASSESSMENT, Fight, FLIGHT, BUT NOT FREEZE IN BLANCHARD'S THREAT SCENARIOS
}

\author{
Dino Krupić1 \& Bojana Dinić² \\ ${ }^{1}$ Faculty of Humanities and Social Sciences, University of Osijek, Osijek, Croatia. \\ ${ }^{2}$ Faculty of Philosophy, University of Novi Sad, Novi Sad, Serbia.
}

\section{dkrupic@ffos.hr}

\begin{abstract}
One of the most frequently used self-report instruments of human defensive behaviors is Blanchards' threat scenarios. The aim of this study is to reevaluate situational cues on defensive behavioral repertoire by the modified version of the instrument. Instead of the first choice response format, we implemented a five-point Likert type scale. This allowed the use of different statistical procedures to reevaluate the instrument. The instrument was administered to 1136 ( $40.85 \%$ males) participants from the general population of Croatia. Results revealed that the scenarios represent valid measures of the risk assessment, fight and flight, but not freezing responses. In addition, it was found that females tend to avoid, while males tend to confront the source of threat. The results are highly congruent with the findings in other cultures. We conclude that this instrument may represent a very useful tool in the research of human defensive behavioral repertoire, except for freeze reactions.
\end{abstract}

Keywords: Threat scenarios, fight, flight, freeze, risk assessment.

\section{INTRODUCTION}

Mammals' defensive behavioral repertoire contains a set of specific behaviors, such as risk assessment, fight, flight and freezing. Numerous studies on rodents have shown that neuropsychological basis for these behaviors is located in lower brain structures (Fanselow, 1994), and it is assumed that they are reflexive behaviors triggered by specific environmental cues (e.g. Adolphs, 2013; Blanchard \& Blanchard, 2008; Bracha, 2004; McNaughton \& Corr, 2008). 
Neurophysiological studies of human defensive system raise numerous ethical concerns, which makes animal studies very valuable in this field. Reasonably, we cannot observe and measure the defensive behavioral repertoire in humans by using the same methods as in animal studies. Hence the study of human defensive behavior frequently relies on self-report instruments, among which the threat scenarios, developed by Blanchard, Hynd, Minke, Minemoto and Blanchard (2001), is the most used one.

Studies conducted with this instrument in Hawaii (Blanchard et al., 2001), Brazil (Shuhama, Del-Ben, Loureiro, \& Graeff, 2008), and UK (Perkins \& Corr, 2006) have shown almost zero cultural effect. This is understandable, since the defensive behaviors should be almost reflexive and deeply biologically grounded (Adolphs, 2013; Canteras \& Blanchard, 2008). All three abovementioned studies show that defensive reactions are triggered by contextual cues. Specifically, ambiguous threat situations tend to elicit risk assessment (Blanchard, Blanchard, \& Rodgers, 1991), while in clearly dangerous situations, fast avoidance response, such as flight, freeze or defensive fight are more appropriate (Bracha, 2004). In addition, flight is more appropriate in situations in which clearly dangerous or alarming stimuli are very distant, because it enables escape. However, if the stimulus is in close spatio-temporal proximity, but the predator has not yet perceived the prey, tonic immobility or freezing serves as a mechanism of avoiding the predator's attention (Schmidt, Richey, Zvolensky, \& Maner, 2008). If the predator is very near and has spotted the prey, i.e. it cannot escape nor "pretend dead" anymore, attacking the predator is the only option for the prey (McNaughton \& Corr, 2008). Table 1 summarize contextual cues of each of defensive behaviors.

Table 1: Summary table explaining type of situation that elicits certain defensive behavior.

\begin{tabular}{lcccc}
\hline & Presence on threat & $\begin{array}{c}\text { Level of } \\
\text { dangerousness }\end{array}$ & Escape & $\begin{array}{c}\text { Possibility to avoid } \\
\text { confrontation with } \\
\text { the threat }\end{array}$ \\
\hline $\begin{array}{l}\text { Risk } \\
\text { Assessment }\end{array}$ & Ambiguity & Low/moderate & Available & Available \\
Flight & Clear & Moderate/high & Available & Available \\
Fight & Clear & High & Not available & Available \\
\hline
\end{tabular}

The aim of this study is to validate Blanchard et al's (2001) threat scenarios with modified response format, which would allow the use of parametric statistics. Although the threat scenarios are frequently used to study human defensive behavioral repertoire (e.g. Krupić, Križanić, \& Corr, 2016; Perkins \& Corr, 2006; Shuhama, et al., 2008), this instrument has not yet been analyzed with parametric statistics procedures, since the original version of the scenarios contains first choice format that provides data on a nominal scale. For the purpose of this study, we will reevaluate the findings yielded by 
the instrument in previous studies by using Likert type answer format instead of the first choice one.

We expect to confirm that the scenarios can be grouped in four situational contexts which elicit certain defensive behavior: (a) risk assessment should be elicited in ambiguous situations; (b) flight response in clearly dangerous, but escapable situations; (c) freeze in clearly dangerous and unescapable situations; and (d) fight in clearly dangerous situations when fight and freeze are not an option anymore (see Table 1). For further validation of modified Blanchard et al's (2011) threat scenarios, we will explore sex differences in defensive behaviors. We expect that males will show a greater tendency to confront the source of threat in comparison to females, who will show a greater tendency to avoid the threat. According to Taylor et al. (2000), the sex differences are a result of differences in the functioning of the neuroendocrine systems. Whereas males tend to engage in more testosterone associated, aggressive behaviors, females tend to have higher level of oxytocin and estrogens that promote non-aggressive behaviors such as tend and befriend.

\section{METHOD}

\section{Participants}

Total of 1136 participants from the general population of Croatia ( $40.85 \%$ male), aged between 12 and $69(M=23.65, S D=8.07)$, completed the threat scenarios with modified response format. The participants were recruited into the study via Limesurvey online survey system that was shared by social networks and local web portals.

\section{Measure and Procedure}

Threat scenarios (Blanchard et al., 2001) was designed to measure ten defensive behaviors in twelve threatening situations. The first group of items (defensive behavior) presents a list of ten behaviors from which participants, in the original instrument, must choose the most likely behavior (i.e. first-choice method) for each of the twelve threat scenarios: hide; freeze, immobilization; run away, try to escape; threaten to scream or call for help; yell, scream, or call for help; threaten to attack; attack or struggle; check out, approach, or investigate; look for something to use as a weapon; and beg, plead for mercy, or negotiate. In this study, besides the first choice, all the defensive behaviors within the scenarios were rated on a Likert scale ranging from $1=$ not likely at all to $5=$ certainly. The second group of items focuses on the description or the perception of the scenario (scenario perception). These items measure: magnitude of threat; escapability of the situation; ambiguity of the threat stimulus; distance between the threat and the subject; presence of a hiding place. These items were rated on a Likert scale ranging from $1=$ not at all to $5=$ extremely. Items in the instrument were presented in consistent order. For each scenario, participants rated their first choice defensive reaction, then the likelihood of all ten defensive reactions (defensive behavior) and the scenario perception items. The same procedure was repeated for each scenario.

The Ethics Committee of the Faculty of Humanities and Social Sciences in Rijeka gave approval for the study. 


\section{RESULTS}

The effect of the situation on human defensive behavior was tested by two repeated ANOVAs. Since Mauchly's spheracity test indicated unequal variances in both analyses, we interpreted the $F$ ratios with Greenhouse-Geisser correction, and Bonferroni as the post hoc pairwise comparison.

In the first repeated ANOVA, five items describing threat scenarios were treated as dependent variables (scenarios perception) and twelve scenarios as independent variables. The results have shown strong differences in perception between the scenarios $\left(F(8.78,9969.55)=386.13, p<.01, \eta_{\mathrm{p}}^{2}=.25\right)$. Figure 1. summarizes the descriptive statistics. Based on Bonferroni post-hoc test (full table can be provided on request), acquaintance and grab scenarios (see Appendix for description) were perceived as ambiguous threat situations where potential threat was in close personal proximity, and thus there was limited possibility to hide, conceal or protect. We labeled this group of scenarios as Psychological risk assessment. Noise, phone, bomb and whisper scenarios were perceived as ambiguous threat situations with possibility to escape or hide. This group of risk-assessment provoking scenarios was labeled as Physical risk assessment. The only scenario that was perceived as clearly dangerous scenario without possibility to freeze or escape, which corresponds to the fight-provoking scenario (Fight), was the elevator scenario. Bush, corner and park scenarios were perceived as clearly (unambiguously) dangerous scenarios with possibility to escape and hence represent the group of flightprovoking scenarios (High flight). Finally, the fifth group of scenarios that encompasses stoplights and tailgating was also perceived as flight-provoking, but with moderate level of dangerousness and thus was labeled as moderate-flight-provoking scenarios (Moderate flight).

Additionally, we tested sex differences in defensive behavioral repertoire across five groups of scenarios with the second repeated ANOVA. The scores of defensive behaviors in groups were averaged. The results have shown significant differences between sexes in defensive reactions across all five groups of threat scenarios (three-way interaction between sex, scenarios and defensive behaviors is significant, $F(36,1099)=18.85, p<$. $\left.01, \eta_{\mathrm{p}}{ }^{2}=.38\right)$. Descriptive statistics are presented in Table 2 .

In general, males are more likely to display more aggressive behavior toward the threat (threaten to attack, attack, look for a weapon) and to explore the dangerous situation (risk assessment), while females tend to avoid danger (hide, freeze, run, threaten to scream, and $y e l l /$ scream). These findings are consistent across groups of threat scenarios, but they are most obvious in fight and flight groups of scenarios. The only non-significant differences were found for hide in Moderate flight and look for a weapon in Psychological and Physical risk assessment, and Fight. Furthermore, there are differences between the sexes in the most likely behavioral reactions in different groups of threat scenarios. The clearest sex difference was found in fight-provoking scenario, in which males were most likely to attack the source of danger, while females were most likely to yell or scream for help. In High flight-provoking situation, the most common male reactions were looking for a weapon and run, while run was the most common one for females. In Moderate flight situation males were more likely to react with run and risk assessment, while females were again more likely to react with just run from the situation. Similarity between sexes 
Table 2: Gender differences in behavioral reactions across five groups of threat scenarios

\begin{tabular}{|c|c|c|c|c|c|c|c|c|c|c|c|c|c|c|c|c|c|c|c|c|}
\hline & \multicolumn{4}{|c|}{ Psychological risk assessment } & \multicolumn{4}{|c|}{ Physical risk assessment } & \multicolumn{4}{|c|}{ Fight } & \multicolumn{4}{|c|}{ Flight } & \multicolumn{4}{|c|}{ Moderate flight } \\
\hline & \multicolumn{2}{|c|}{ Male } & \multicolumn{2}{|c|}{ Female } & \multicolumn{2}{|c|}{ Male } & \multicolumn{2}{|c|}{ Female } & \multicolumn{2}{|c|}{ Male } & \multicolumn{2}{|c|}{ Female } & \multicolumn{2}{|c|}{ Male } & \multicolumn{2}{|c|}{ Female } & \multicolumn{2}{|c|}{ Male } & \multicolumn{2}{|c|}{ Female } \\
\hline & M & SD & M & SD & M & SD & M & SD & M & SD & M & SD & M & SD & M & SD & M & SD & M & SD \\
\hline Hide & 1.34 & 0.62 & 1.48 & 0.68 & 1.87 & 0.87 & 2.59 & 0.98 & 1.35 & 0.70 & 1.51 & 0.86 & 1.87 & 0.78 & 2.40 & 0.88 & 1.37 & 0.68 & 1.41 & 0.66 \\
\hline Freeze & 1.75 & 0.86 & 2.47 & 0.97 & 1.84 & 0.92 & 2.82 & 1.10 & 1.90 & 1.00 & 2.77 & 1.23 & 1.83 & 0.86 & 2.56 & 0.98 & 1.55 & 0.77 & 2.04 & 0.93 \\
\hline Run & 2.14 & 0.98 & 2.91 & 0.97 & 2.06 & 0.84 & 2.68 & 0.93 & 2.45 & 1.24 & 3.58 & 1.23 & 3.16 & 1.07 & 4.21 & 0.73 & 2.95 & 1.28 & 3.93 & 1.02 \\
\hline $\begin{array}{l}\text { Threaten to } \\
\text { Screem }\end{array}$ & 1.77 & 0.87 & 2.59 & 1.04 & 1.72 & 0.79 & 2.09 & 0.87 & 2.15 & 1.13 & 3.55 & 1.19 & 2.10 & 0.89 & 3.17 & 1.00 & 1.73 & 0.87 & 2.37 & 0.97 \\
\hline Yell scream & 1.80 & 0.91 & 2.66 & 0.96 & 1.88 & 0.89 & 2.40 & 0.94 & 2.37 & 1.24 & 4.08 & 1.07 & 2.23 & 0.95 & 3.59 & 0.88 & 1.71 & 0.90 & 2.35 & 0.99 \\
\hline $\begin{array}{l}\text { Threaten to } \\
\text { attack }\end{array}$ & 2.67 & 1.08 & 2.42 & 1.05 & 2.12 & 0.89 & 1.80 & 0.77 & 3.30 & 1.20 & 2.84 & 1.30 & 2.86 & 0.97 & 2.30 & 0.93 & 2.72 & 1.09 & 2.23 & 1.07 \\
\hline Attack & 2.67 & 1.03 & 2.49 & 1.01 & 2.22 & 0.89 & 1.85 & 0.78 & 3.78 & 1.14 & 3.28 & 1.30 & 3.01 & 0.96 & 2.43 & 0.95 & 2.63 & 1.07 & 2.03 & 0.99 \\
\hline $\begin{array}{l}\text { Risk } \\
\text { assessment }\end{array}$ & 3.68 & 1.13 & 3.37 & 1.12 & 3.94 & 0.92 & 3.65 & 1.01 & 2.97 & 1.26 & 2.32 & 1.24 & 2.92 & 1.02 & 2.11 & 0.96 & 3.01 & 1.22 & 2.39 & 1.13 \\
\hline $\begin{array}{l}\text { Look for a } \\
\text { weapon }\end{array}$ & 2.41 & 1.08 & 2.31 & 1.03 & 2.99 & 0.97 & 3.11 & 0.95 & 3.27 & 1.25 & 3.22 & 1.28 & 3.31 & 1.00 & 3.11 & 1.00 & 2.56 & 1.12 & 2.25 & 1.05 \\
\hline $\begin{array}{l}\text { Beg plead } \\
\text { negotiate }\end{array}$ & 1.74 & 0.91 & 1.97 & 0.98 & 1.55 & 0.77 & 1.73 & 0.82 & 1.97 & 1.05 & 2.56 & 1.18 & 1.85 & 0.84 & 2.24 & 0.95 & 1.54 & 0.80 & 1.64 & 0.81 \\
\hline
\end{tabular}

Note: bolded scores are the most probable defensive reaction in certain type of threat situation 
was seen in Psychological and Physical risk assessment scenarios in which both sexes were most likely to investigate the potential danger (risk assessment).

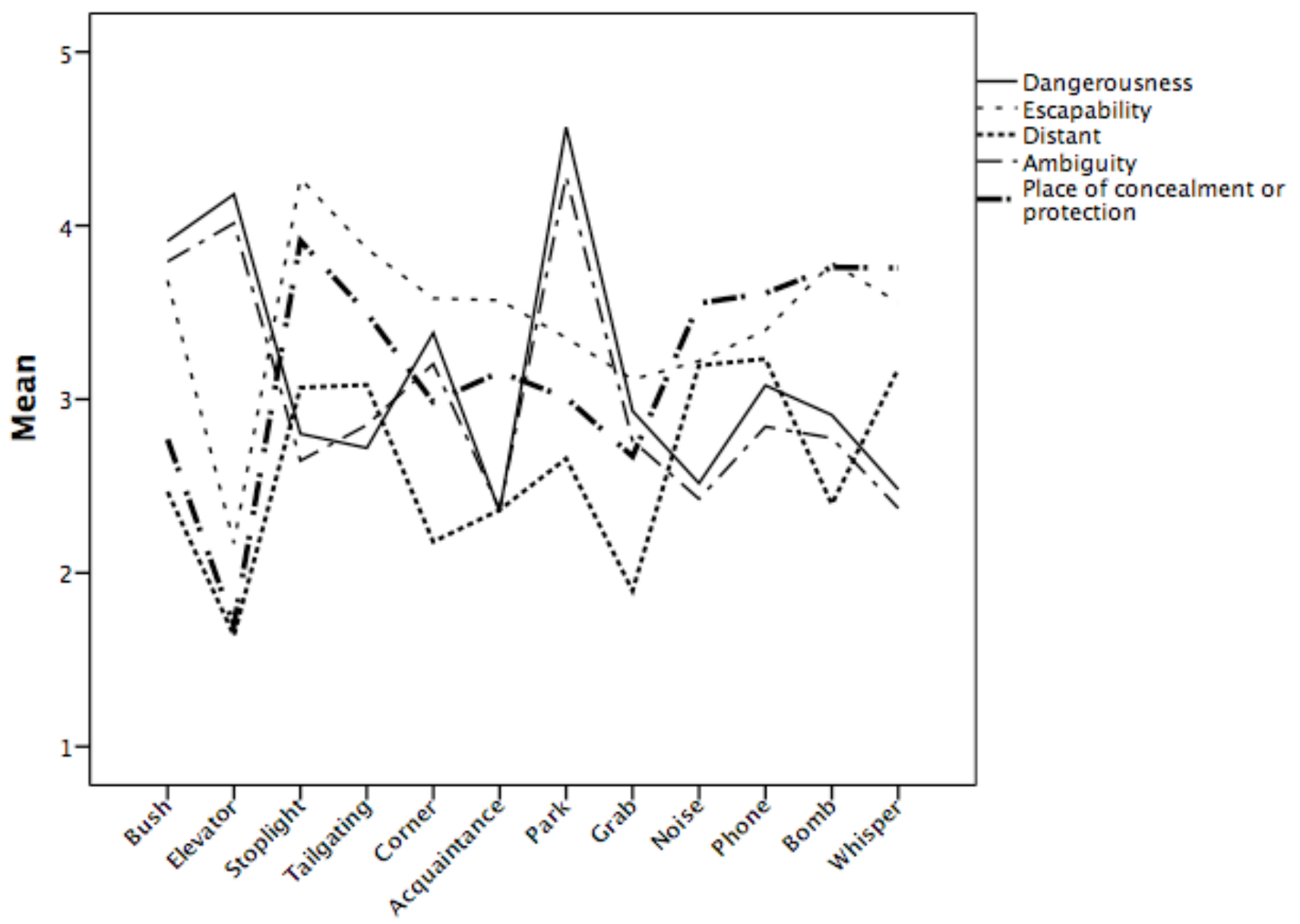

Figure 1: Means of threat scenarios based on five items of scenario perception.

\section{DISCUSSION}

The aim of this study was to examine the effect of situations' perception on human defensive behavior using the threat scenarios (Blanchard et al., 2001). According to Blanchard et al. (2001), four groups of threat scenarios were expected (risk assessment, fight, flight and freeze). On contrary, the results of this study suggested five groups Physical and Psychological risk assessment, Fight, Moderate flight and High flight scenarios. The main finding was that the instrument does not contain a scenario that might provoke freeze behavior.

One of the reasons why freeze scenarios' group was not identified could be the inappropriateness of self-report methodology to asses such type of behavior. Namely, Volchan et al. (2017) found that different types of freezing last much shorter in comparison to other behaviors, which could be the reason for the lower awareness of the freeze reactions in participants. The second reason might be that all scenarios include 
another human as a potential threat. It is possible that threats including animals are more likely to produce freeze reaction. Our data are in line with Harrison, Ahn and Adolphs (2015) results showing that freeze was never the most common response in the original Blanchard scenarios, but it was the most common one for the animal-threat scenario (see Harrison et al., 2015). Nevertheless, the current version of the instrument simply does not have a freeze-provoking scenario, which supports the importance of new freeze inducing scenarios for the study of freeze reactions, which was done in Harrison et al's (2015).

Furthermore, results regarding the two types of risk assessments are also in line with Harrison et al.'s (2015) distinction between physical (dangerous animals or environmental disasters) and psychological (interaction with another person) types of risk assessments in humans. Although the common feature of both risk assessment scenarios is a higher level of ambiguity (Blanchard, Griebel, Pobbe, \& Blanchard, 2011), they can differ in the defensive reaction (see Harrison et al., 2015). Our data indicates that Blanchard's threat scenarios can also be used to assess both types of risk assessment. Differentia specifica of these two groups of scenarios is the presence of a person, where scenarios involving another person as a potential source of threat represent Psychological risk-assessment scenario. Nevertheless, the results from Table 2 prove that both groups of scenarios evoke risk-assessment.

The fight provoking scenarios are represented by only one scenario (the elevator), where danger is clearly present and intense, and there is no place to escape. Finally, the two flight provoking scenarios differ in defensive intensity or dangerousness of the threat, but both are characterized by clear presence of danger with the possibility to escape.

To further examine situational threat scenarios' effect on defensive behavior, we tested sex differences in defensive reactions. In general, females tend to avoid the source of threat, while males are more prone to confronting it, which is congruent with findings of Blanchard et al. (2001), and Harrison et al. (2015). Females tend to scream/yell for a help and run away in dangerous situations, while males are more prone to attacking/ threatening to attack and looking for a weapon, which is also congruent with the findings of Taylor et al. (2000). Namely, these sex differences are the result of different neurobiology and the environment of evolutionary adaptedness. While males tend to have more testosterone associated, aggressive behaviors, females tend to have higher level of oxytocin and estrogens that promote non-aggressive behaviors such as tend and befriend. Furthermore, since the defensive fight reactions elevate the potential threat for women during pregnancy, their defensive behavioral repertoire is more oriented towards avoiding any source of threat in the first place, rather than exposing themselves to dangerous threats by confronting them (Taylor et al., 2000).

Finally, the perception of scenarios in Croatian sample did not differ greatly from the studies conducted in Brazil and Hawaii. The UK studies (Perkins et al., 2010, Perkins \& Corr, 2006) did not provide any data on the perception of scenarios, which our cross cultural comparison is based upon. Table 3. summarizes the findings for scenarios' perception from the three studies that contain the results of the perception across scenarios. As it can be seen, the perception of scenarios is highly congruent among cultures, suggesting that there are no significant cultural differences in perceiving threat situations. Nevertheless, direct comparison in cross-cultural studies is warranted. 
However, it is important to emphasize that all abovementioned studies (including this one) were conducted on non-representative samples. In future studies, it would be interesting to assess the defensive behavioral repertoire among participants with different socioeconomic background and place of residence. Namely, some areas of towns and cities are less secure than others. For instance, Brazilian favelas are significantly more dangerous compared to a more touristic route in Rio de Janeiro. To sum up, previous personal experience could have a significant impact on the defensive behavioral repertoire, just as it has on coping strategies in general (e.g. Knežević, Krupić, \& Šućurović, 2016).

Table 3: Comparison of the most salient scenarios rated on five items measuring perception of threat scenarios from samples from Croatia, Brazil and Hawaii

\begin{tabular}{|c|c|c|c|c|}
\hline & & Croatia & $\begin{array}{l}\text { Brazil (Shuhama et } \\
\text { al., 2008) }\end{array}$ & $\begin{array}{l}\text { Hawaii (Blanchard } \\
\text { et al.,2001) }\end{array}$ \\
\hline \multirow[b]{2}{*}{ Dangerousness } & Highest & park, elevator & park, elevator & park \\
\hline & Lowest & $\begin{array}{l}\text { whisper, noise, } \\
\text { acquaintance }\end{array}$ & $\begin{array}{l}\text { whisper, noise, } \\
\text { acquaintance }\end{array}$ & acquaintance \\
\hline \multirow{2}{*}{ Escapability } & Highest & stoplight, tailgating & $\begin{array}{l}\text { stoplight, } \\
\text { tailgating }\end{array}$ & stoplight \\
\hline & Lowest & elevator & elevator & elevator \\
\hline \multirow{2}{*}{ Distant } & Highest & whisper, noise, phone & $\begin{array}{l}\text { whisper, noise, } \\
\text { phone }\end{array}$ & whisper \\
\hline & Lowest & elevator, grab, corner & $\begin{array}{l}\text { elevator, grab, } \\
\text { corner }\end{array}$ & elevator, grab \\
\hline \multirow[t]{2}{*}{ Ambiguity } & Highest & $\begin{array}{l}\text { acquaintance, noise, } \\
\text { whisper }\end{array}$ & $\begin{array}{l}\text { acquaintance, } \\
\text { noise, whisper }\end{array}$ & $\begin{array}{l}\text { acquaintance, } \\
\text { noise }\end{array}$ \\
\hline & Lowest & park & park & park \\
\hline \multirow{2}{*}{$\begin{array}{l}\text { Place of } \\
\text { concealment or } \\
\text { protection }\end{array}$} & Highest & spotlight, whisper & spotlight, whisper & $\begin{array}{l}\text { noise, whisper, } \\
\text { phone }\end{array}$ \\
\hline & Lowest & elevator, grab & elevator, grab & \\
\hline
\end{tabular}

Some methodological concerns should be highlighted before the conclusion. The first choice answer format may seem more ecological than the likelihood estimation of all ten defensive behaviors measured by Likert type answer format. Indeed, out of many potential defensive reactions, an individual can react only in one certain way in any given situation. However, both answer formats have their own weakness and strengths. The contribution of this study is that Blanchard et al.s (2001) threat scenarios were assessed 
by using a different answer format, which produced novel findings regarding the instrument.

To sum up, results indicate which of the scenarios within the instrument are conceptually similar, and which scenarios elicit which defensive reaction. The results of the study suggest that the threat scenarios do not cover whole range of defensive behavior in humans. As a result, Harrison et al.s (2015) version of the instrument represents a promising solution for the study of human defensive behavior.

\section{REFERENCES}

Adolphs, R. (2013). The biology of fear. Current Biology, 23(2), 79-93. DOI

Blanchard, D. C., \& Blanchard, R. J. (2008). Defensive behaviors, fear, and anxiety. In R. J. Blanchard, D. C. Blanchard, G. Griebel, \& D. Nutt (Eds), Handbook of anxiety and fear (Vol. 17, pp.63-80). Amsterdam: Elsevier. DOI

Blanchard, D. C., Griebel, G., Pobbe, R., \& Blanchard, R. J. (2011). Risk assessment as an evolved threat detection and analysis process. Neuroscience \& Biobehavioral Reviews, 35(4), 991-998. DOI

Blanchard, D. C., Hynd, A. L., Minke, K. A., Minemoto, T., \& Blanchard, R. J. (2001). Human defensive behaviors to threat scenarios show parallels to fear-and anxiety-related defense patterns of non-human mammals. Neuroscience and Biobehavioral Reviews, 25(7), 761-770. DOI

Bracha, H. S. (2004). Freeze, flight, fight, fright, faint: Adaptationist perspectives on the acute stress response spectrum. CNS Spectrums, 9(9), 679-685. DOI

Canteras, N. S., \& Blanchard, D. C. (2008). A behavioral and neural systems comparison of unconditioned and conditioned defensive behavior. In R. J. Blanchard, D. C. Blanchard, G. Griebel, \& D. Nutt (Eds.), Handbook of anxiety and fear (pp. 141-153). Elsevier. DOI

Fanselow, M. S. (1994). Neural organization of the defensive behavior system responsible for fear. Psychonomic Bulletin \& Review, 1(4), 429-438. DOI

Harrison, L. A., Ahn, C., \& Adolphs, R. (2015). Exploring the structure of human defensive responses from judgments of threat scenarios. PloS One, 10(8), e0133682. DOI

Knežević, M., Krupić, D., \& Šučurović, S. (2016). Coping strategies in war veterans 20 years after the exposure to extreme stress. Journal for General Social Issues, 25(3), 353-370.

Krupić, D., Križanić, V., Corr, P. J. (2016). Personality and defensive behaviour: A factor analytic approach to threat scenario choices. Personality and Individual Differences, 94, 303-308. DOI

McNaughton, N., \& Corr, P. J. (2008). The neuropsychology of fear and anxiety: A foundation for reinforcement sensitivity theory. In P. J. Corr (Ed.), The reinforcement sensitivity theory of personality (pp. 44-94). Cambridge: Cambridge University Press. DOI

Perkins, A. M., \& Corr, P. J. (2006). Reactions to threat and personality: Psychometric differentiation of intensity and direction dimensions of human defensive behaviour. Behavioural Brain Research, 169(1), 21-28. DOI 
Schmidt, N. B., Richey, J. A., Zvolensky, M. J., \& Maner, J. K. (2008). Exploring human freeze responses to a threat stressor. Journal of Behavior Therapy and Experimental Psychiatry, 39(3), 292-304. DOI

Shuhama, R., Del-Ben, C. M., Loureiro, S. R., \& Graeff, F. G. (2008). Defensive responses to threat scenarios in Brazilians reproduce the pattern of Hawaiian Americans and nonhuman mammals. Brazilian Journal of Medical and Biological Research, 41(4), 324-332. $\underline{\mathrm{DOI}}$

Taylor, S. E., Klein, L. C., Lewis, B. P., Gruenewald, T. L., Gurung, R. A., \& Updegraff, J. A. (2000). Biobehavioral responses to stress in females: tend-and-befriend, not fight-orflight. Psychological Review, 107(3), 411-429. DOI

Volchan, E., Rocha-Rego, V., Bastos, A. F., Oliveira, J. M., Franklin, C., Gleiser, S., ... \& Erthal, F. S. (2017). Immobility reactions under threat: A contribution to human defensive cascade and PTSD. Neuroscience and Biobehavioral Reviews. In Press. DOI 